\title{
Identification of susceptibility loci for cardiovascular disease in adults with hypertension, diabetes, and dyslipidemia
}

Youhyun Song ${ }^{1 \dagger}$, Ja-Eun Choi ${ }^{2 \dagger}$, Yu-Jin Kwon ${ }^{3}$, Hyuk-Jae Chang ${ }^{4}$, Jung Oh Kim², Da-Hyun Park ${ }^{2}$, Jae-Min Park', Seong-Jin Kim², Ji Won Lee ${ }^{1^{*}}$ (D) and Kyung-Won Hong ${ }^{2^{*}}$

\begin{abstract}
Background: Hypertension (HTN), diabetes mellitus (DM), and dyslipidemia (DL) are well-known risk factors of cardiovascular disease (CVD), but not all patients develop CVDs. Studies have been limited investigating genetic risk of CVDs specific to individuals with metabolic diseases. This study aimed to identify disease-specific and/or common genetic loci associated with CVD susceptibility in chronic metabolic disease patients.

Methods: We conducted a genome-wide association study (GWAS) of a multiple case-control design with data from the City Cohort within Health EXAminees subcohort of the Korean Genome and Epidemiology Study (KoGES_HEXA). KoGES_HEXA is a population-based prospective cohort of 173,357 urban Korean adults that had health examinations at medical centers. 42,393 participants (16,309 HTN; 5,314 DM; 20,770 DL) were analyzed, and each metabolic disease group was divided into three CVD case-controls: coronary artery disease (CAD), ischemic stroke (IS), and cardio-cerebrovascular disease (CCD). GWASs were conducted for each case-control group with 7,975,321 imputed single nucleotide polymorphisms using the Phase 3 Asian panel from 1000 Genomes Project, by logistic regression and controlled for confounding variables. Genome-wide significant levels were implemented to identify important susceptibility loci.

Results: Totaling 42,393 individuals, this study included 16,309 HTN (mean age [SD], 57.28 [7.45]; 816 CAD, 398 IS, and 1,185 CCD cases), 5,314 DM (57.79 [7.39]; 361 CAD, 153 IS, and 497 CCD cases), and 20,770 DL patients (55.34 [7.63]; 768 CAD, 295 IS, and 1,039 CCD cases). Six genome-wide significant CVD risk loci were identified, with relatively large effect sizes: 1 locus in HTN (HTN-CAD: 17q25.3/CBX8-CBX4 [OR, 2.607; $\left.P=6.37 \times 10^{-9}\right]$ ), 2 in DM (DM-IS: 4q32.3/MARCH1-LINC01207 [OR, 5.587; $\left.P=1.34 \times 10^{-8}\right]$, and DM-CCD: 17q25.3/RPTOR [OR, 3.511; $\left.P=1.99 \times 10^{-8}\right]$ ), and 3 in DL (DL-CAD: 9q22.2/UNQ6494-LOC101927847 [OR, 2.282; $P=7.78 \times 10^{-9}$ ], DL-IS: 3p22.1/ULK4 [OR, 2.162; $\left.P=2.97 \times 10^{-8}\right]$, and DL-CCD: 2p22.2/CYP1B1-CYP1B1-AS1 [OR, 2.027; $\left.P=4.24 \times 10^{-8}\right]$ ).
\end{abstract}

\footnotetext{
*Correspondence: indi5645@yuhs.ac; kyungwon.hong@theragenbio.com

${ }^{\dagger}$ Youhyun Song and Ja-Eun Choi are co-first authors who equally

contributed to this work.

${ }^{1}$ Department of Family Medicine, Gangnam Severance Hospital, Yonsei

University College of Medicine, 211, Eonju-ro, Gangnam-gu, Seoul 06273,

Korea

2 Healthcare R\&D Division, Theragen Bio Co., Ltd., Gwanggyo-ro 145,

Suwon-si, Gyeonggi-do 16229, Republic of Korea

Full list of author information is available at the end of the article
}

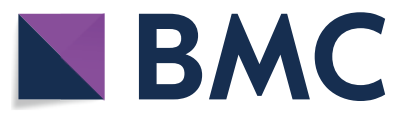

(c) The Author(s) 2021. This article is licensed under a Creative Commons Attribution 4.0 International License, which permits use, sharing, adaptation, distribution and reproduction in any medium or format, as long as you give appropriate credit to the original author(s) and the source, provide a link to the Creative Commons licence, and indicate if changes were made. The images or other third party material in this article are included in the article's Creative Commons licence, unless indicated otherwise in a credit line to the material. If material is not included in the article's Creative Commons licence and your intended use is not permitted by statutory regulation or exceeds the permitted use, you will need to obtain permission directly from the copyright holder. To view a copy of this licence, visit http://creativeco mmons.org/licenses/by/4.0/. The Creative Commons Public Domain Dedication waiver (http://creativecommons.org/publicdomain/ zero/1.0/) applies to the data made available in this article, unless otherwise stated in a credit line to the data. 
Conclusions: This study identified 6 susceptibility loci and positional candidate genes for CVDs in HTN, DM, and DL patients using an unprecedented study design. 1 locus (17q25.3) was commonly associated with CAD. These associations warrant validation in additional studies for potential therapeutic applications.

Keywords: Hypertension, Diabetes mellitus, Dyslipidemia, Cardiovascular disease, Genome-wide association studies

\section{Background}

Cardiovascular disease (CVD) is the leading cause of death globally, and the prevalence of cardiovascular disease is constantly progressing in both developed and developing nations [1]. According to a World Health Organization report, an estimated 17.9 million people died from heart disease and stroke in 2016, representing $31 \%$ of all global deaths, and it was projected that about 23.6 million people will die from CVDs by 2030 [2]. In line with the global trend, economic and social developments have significantly impacted lifestyles of Koreans; CVDs are now one of the main causes of death in Korea, accounting for 1 in every 5 deaths [3]. Therefore, interventions to prevent and reduce cardiovascular risk factors are warranted.

Epidemiological evidence suggest that CVD is associated with age, sex, ethnicity, behavioral risk factors (smoking, alcohol use, and low physical activity levels), and several chronic metabolic diseases. Among them, hypertension (HTN), dyslipidemia (DL), and type 2 diabetes (DM) are the most common modifiable risk factors for CVD, which is known as the most prevalent cause of mortality and morbidity in populations with HTN, DM, and DL [4]. Approximately two-thirds of all adults with HTN at 30 years of age have a $\sim 40 \%$ higher risk of experiencing a CVD event and systematic reviews have revealed that lowering blood pressure greatly reduced major CVD events and all-cause mortality, irrespective of the initial blood pressure [5]. Patients with DM had a $10 \%$ greater risk of coronary artery disease (CAD), a $53 \%$ greater risk of myocardial infarction (MI), and a $58 \%$ greater risk of stroke compared to those without diagnoses of DM, and CVD accounts for approximately half of all deaths in patients with DM, largely due to an increased risk of stroke and MI [6]. Further, in the INTERHEART study, $49 \%$ of the population-attributable risk of a first MI was contributed by DL, and the prevalence of DL was found to be between 83 and $87 \%$ in Asian patients with CVD [7].

There are various biochemical mechanisms that independently increase the risk of CVD in people with these chronic metabolic diseases. Lipid oxidation is thought to be an important determinant of atherosclerosis, which leads to CVD; moreover, high levels of low-density lipoprotein (LDL) and low levels of high-density lipoprotein (HDL) are associated with MI and stroke in DL [8].
Endothelial dysfunction, vascular inflammation, arterial remodeling, atherosclerosis, dyslipidemia, and obesity are common risk factors for CVD in HTN and DM. In addition, upregulation of the renin-angiotensin-aldosterone system, oxidative stress, inflammation, and activation of the immune system contribute to the close relationship between DM, HTN, and CVD [9].

However, only limited evidence is available regarding the relationship between multiple chronic metabolic diseases and CVDs. Furthermore, despite maintaining optimal levels of metabolic indices, such as blood pressure, glucose, or lipids, CVDs still remain prevalent in individuals with HTN, DM, and DL. Evidence suggest that although hyperglycemia contributes to ischemic events, it is not the only factor, because both pre-diabetes and normoglycemic patients show risk for most types of CVD [10]. High-dose statin therapy may provide some incremental benefit between 10 and 20\%; nevertheless, statin-treated patients remain at high residual risk for future cardiovascular events [11]. This suggests that other non-modifiable risk factors, such as genetic and genomic variables, need to be considered as well.

Genetic factors are important contributors to the risk of CVDs; recent genome-wide association studies (GWASs) strongly support that genetic susceptibility to CVD is largely derived from gene mutations or variation [12]. Loci nearest a lead single nucleotide polymorphism (SNP) was shown to have genome-wide significant associations with obesity, blood pressure, lipids, DM, CAD, and stroke [13]. However, over the past decade, the majority of GWASs for CVDs or related risk factors have been conducted in populations with European white ancestry and limited evidence is available in Asian populations [14]. Furthermore, few analyses focused on the genetic differences in CVD susceptibility between individuals with the same chronic metabolic conditions.

Therefore, we conducted three sets of GWASs between case-controls of major CVDs in HTN, DM, and DL patient groups using data from the well-known nationwide Korean Genome and Epidemiology Study (KoGES). We aimed to find genetic pathways linking the development of CVD with each metabolic disease group; we further aimed to identify the potential presence of a common pathway leading to CVD among the commonly co-existing metabolic conditions. 


\section{Methods}

\section{Study populations}

Our study used a part of the KoGES dataset obtained from the Korean Center for Disease Control and Prevention. The largest cohort of KoGES is the health examination cohort [KoGES_HEXA], and its dataset consists of participants' medico-pharmacologic history, anthropometric traits, and blood biochemistry traits [15]. Briefly, KoGES_HEXA is a population-based prospective cohort of 173,357 urban Korean adults that had health examinations at medical centers, recruited from the national health examinee registry. Participants were men and women, aged 40-69 years from 14 major cities across Korea and recruited at baseline between 2004 and 2013. A total of 58,701 participants, whose genome-wide SNP genotype data were obtained, were included in the citybased cohort of the KoGES. All participants voluntarily signed an informed consent form before the study, and the study protocol was approved by the Institutional Review Boards (IRB) of the institutions that participated in KoGES. This study was performed in accordance with the Declaration of Helsinki and approved by the IRB of Theragen Etex (Approval Numbers: 700062-20190819-GP-006-02).

\section{Measurement of anthropometric and laboratory data \& Definition of lifestyle factors}

Study participants completed a standardized medical history and lifestyle questionnaire and underwent a comprehensive health examination by trained medical staff according to a standard protocol. Smoking status was classified into three groups: participants who had smoked over 100 cigarettes throughout their lifetimes but had quit before this study were ex-smokers, currently smoking were current smokers, and the remaining were non-smokers. Drinking status (alcohol intake) was also classified into three groups: current drinkers, ex-drinkers, and non-drinkers. Regularity of physical activity was determined according to whether subjects participated regularly in any sports to the point of sweating.

Body mass index (BMI) was calculated as weight in kilograms divided by height in meters squared $\left(\mathrm{kg} / \mathrm{m}^{2}\right)$. Systolic (SBP) and diastolic blood pressure (DBP) were measured twice by a standardized mercury sphygmomanometer (Baumanometer-Standby; W.A. Baum Co. Inc., New York, NY, USA). Blood samples were drawn after overnight fasting, and venous blood sampling was collected in a plain tube. Biochemical parameters, including fasting glucose, hemoglobin A1c (HbA1c), total cholesterol, HDL cholesterol, and triglycerides (TG), were determined by enzymatic methods (ADVIA 1650, Siemens, Tarrytown. NY, USA). LDL- cholesterol was calculated using the Friedewald equation
(LDL-cholesterol $=$ total cholesterol - HDL- cholesterol $-[\mathrm{TG} / 5])$.

\section{Definition of study phenotypes}

HTN was defined as systolic $\mathrm{BP} \geq 140 \mathrm{mmHg}$ or diastolic $\mathrm{BP} \geq 90 \mathrm{mmHg}$ on health examination, currently taking an anti-hypertensive drug, or diagnosed by a physician. DM was defined as fasting blood glucose $\geq 126 \mathrm{mg} / \mathrm{dl}$, $\mathrm{HbA} 1 \mathrm{c} \geq 6.5 \%(48 \mathrm{mmol} / \mathrm{mol})$, currently taking an antidiabetic drug or insulin, or diagnosed by a physician.

DL was defined as either diagnosis by a physician, current use of lipid-lowering medication, or according to the National Cholesterol Education ProgramAdult Treatment Panel III (NCEP-ATP III) criteria: (1) hypercholesterolemia (serum $\mathrm{TC} \geq 240 \mathrm{mg} / \mathrm{dl}$ ), (2) hypertriglyceridemia (serum TG $\geq 200 \mathrm{mg} / \mathrm{dl}$ ), (3) hyperLDL-cholesterolemia (serum LDL-C $\geq 160 \mathrm{mg} / \mathrm{dl}$ ), (4) hypo-HDL-cholesterolemia (serum HDL-C $<40 \mathrm{mg} / \mathrm{dl}$ ).

\section{Outcome measurements}

We defined CAD as the participant-reported history of the diagnosis or treatment of angina pectoris or myocardial infarction. Ischemic stroke (IS) was defined in the same manner, in that it was based on the participant-reported history of the diagnosis or treatment of ischemic stroke. Cardio-cerebrovascular disease (CCD) was defined as the combination of CAD and IS per our study outcome definition.

\section{Study design}

This study investigated the genetic risk factors of CVDs in the patients with metabolic disease (HTN, DM, or DL). For this analysis, we applied the exclusion criteria schematically illustrated in Fig. 1: from baseline we excluded participants with missing data values, i.e. smoking, alcohol, exercise history and body mass index (BMI) $(n=471)$. Subsequently, participants with histories of malignancy or no response regarding malignancy were excluded $(n=2,202)$. After these exclusions, 56,028 participants were included; the final sample size for the present analysis was 16,313 participants with HTN, 5394 participants with DM, and 20,788 participants with DL.

\section{Genotyping and quality control procedures}

The genotype data were graciously provided by the Center for Genome Science, Korea National Institute of Health. The genotype data was produced by the Korea Biobank Array (Affymetrix, Santa Clara, CA, USA) (Moon et al., 2019, PMID 30718733). The experimental results of Korea Biobank Array were filtered by the quality control procedures of the following criteria: call rate higher than 97\%, minor allele frequency higher than $1 \%$, and Hardy-Weinberg equilibrium test $\mathrm{p}<1 \times 10^{-5}$. 


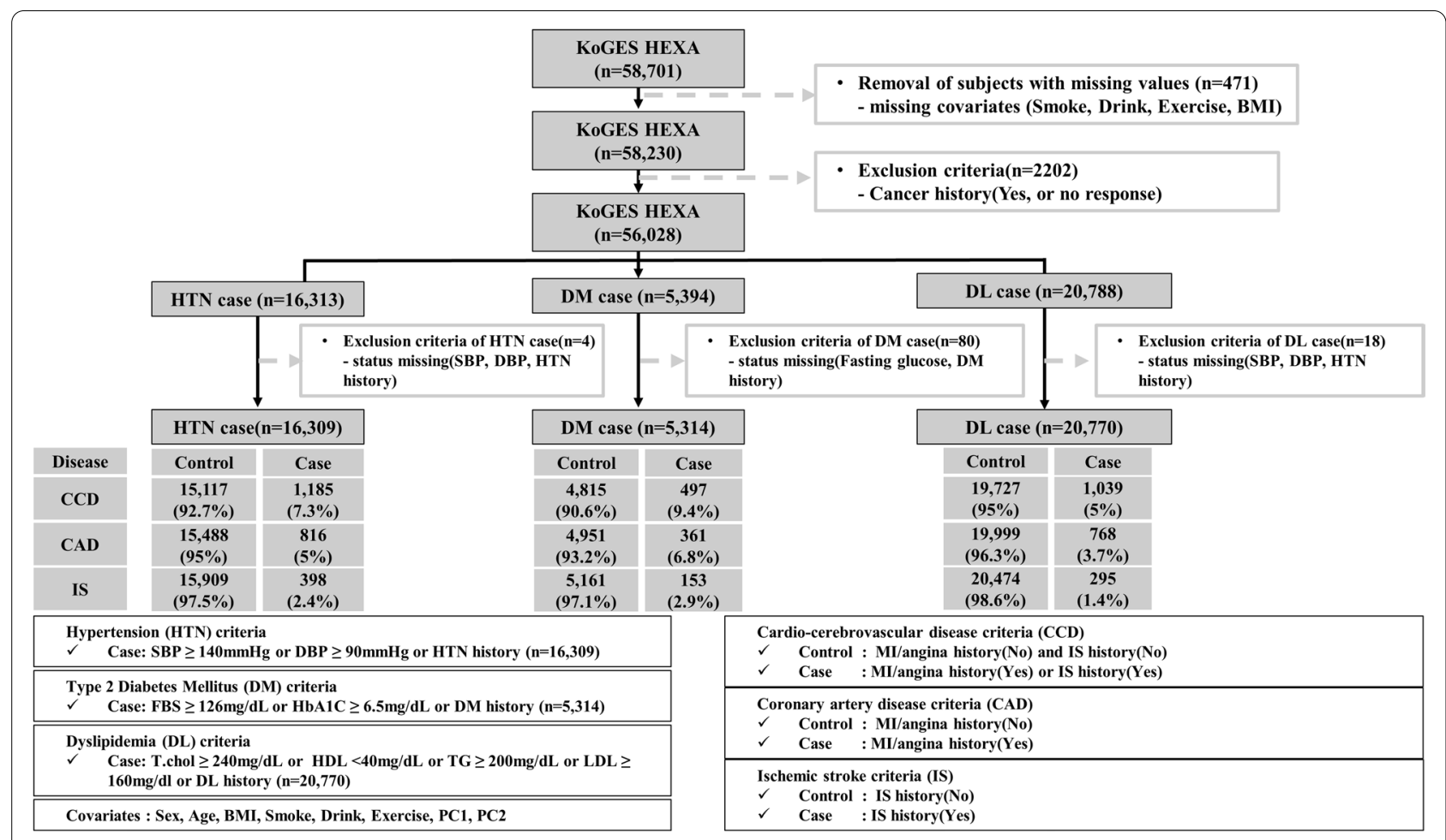

Fig. 1 Study Design. Flow diagram illustrating the study sample selection process and overall study design

After the quality control procedures, the experimental genotypes were phased using ShapeIT v2 and IMPUTE v2 was used for imputation analysis of the phased genotype data with 1000 Genomes Phase3 data as a reference panel. After imputation, imputed variants with imputation quality score $<0.4$ or MAF $<1 \%$ were excluded from further analysis (Moon et al., 2019, PMID 30718733). Finally, the number of SNPs for the GWAS was 7,975,321 SNPs from chromosomes 1 to 22 . We associated the closest, or nearby genes of the significant variants as candidate genes utilizing LocusZoom.

\section{Statistical analysis}

All data are presented as the mean \pm standard deviation (SD), or number (\%). For the discovery GWAS analysis, the association between individual SNP genotypes and CAD, IS, and CCD risks were modeled additively for each copy of the minor allele using logistic regression adjusted for age, sex, BMI, exercise status, smoking status, alcohol intake, and PC1 and PC2 as covariates using PLINK, version 1.9 [16]. PC1 and PC2 were obtained through a principal component analysis, which was conducted to reduce the bias of genomic data due to the regional differences in sample collection. We selected high linkage disequilibrium (LD) and cluster SNPs wherein no SNP gap exceeded $50 \mathrm{~kb}$ with high LD $\left(\mathrm{r}^{2}>0.8\right)$ from the top significant SNPs. The significant associations were defined by genome-wide significance level $p$-values $\left(5.00 \times 10^{-8}\right)$ [17]. The gene-region plot of the top SNP associations was generated with Locus- Zoom version 0.4.8.2 [18].

\section{Results}

Clinical characteristics of the 42,393 individuals included in the GWAS are shown in Table 1, and further details categorized by disease status are in Additional file 1: Tables S1-S3. A total of 16,309 individuals with HTN (56.1\% female and 57.28 [7.45] years old), 5,314 with DM (50.9\% female and 57.79 [7.39] years old), and 20,770 with DL (58.4\% female and 55.34 [7.63] years old) were included; the prevalence of CCD was $7.3 \%$ in HTN, 9.4\% in DM, and 5\% in DL, respectively. Results of the 9 GWASs are depicted in Fig. 2 as Manhattan plots using $\log 10$ transformed p-values. The leading SNPs in association with CAD, IS, and CCD for each group are described in Table 2 . The full lists of genomewide significant $\left(P\right.$-value $\left.<5 \times 10^{-8}\right)$ and suggestive $\left(5 \times 10^{-8} \leq P\right.$-value $\left.<1 \times 10^{-5}\right)$ SNPs from the GWASs are available in Additional file 2: Tables S4-S6.

\section{GWAS results of CVDs in Hypertension}

The GWAS of HTN-CAD (Fig. 2a) showed 1 genomewide significant association locus (17q25.3) and 19 loci of suggestive association, HTN- IS (Fig. 2b) showed 23 suggestive loci, and HTN-CCD (Fig. 2c) showed 
Table 1 Clinical characteristics of the study population

\begin{tabular}{|c|c|c|c|}
\hline Characteristics & HTN & DM & $\mathrm{DL}$ \\
\hline Number of individuals, No. & 16,309 & 5314 & 20,770 \\
\hline Female, No. (\%) & $9146(56.1 \%)$ & 2706 (50.9\%) & $12,125(58.4 \%)$ \\
\hline Age, y & $57.28(7.45)$ & $57.79(7.39)$ & $55.34(7.63)$ \\
\hline Coronary artery disease, №. (\%) & $816(5.0 \%)$ & $361(6.8 \%)$ & $768(3.7 \%)$ \\
\hline Ischemic stroke, No. (\%) & $398(2.4 \%)$ & $153(2.9 \%)$ & $295(1.4 \%)$ \\
\hline $\begin{array}{l}\text { Cardio-cerebrovascular disease, No. } \\
(\%)\end{array}$ & $1185(7.3 \%)$ & $497(9.4 \%)$ & $1039(5.0 \%)$ \\
\hline \multicolumn{4}{|l|}{ Anthropometric traits } \\
\hline Body mass index, $\mathrm{kg} / \mathrm{m}^{2}$ & $25.04(2.95)$ & $25.12(3.07)$ & $24.68(2.82)$ \\
\hline Waist circumference, cm & $84.37(8.38)$ & $85.52(8.44)$ & $83.37(8.28)$ \\
\hline Systolic blood pressure, $\mathrm{mmHg}$ & $135.1(14.58)$ & $127.55(14.93)$ & $125.07(14.64)$ \\
\hline Diastolic blood pressure, $\mathrm{mmHg}$ & $83.2(9.88)$ & $77.48(9.37)$ & $77.33(9.61)$ \\
\hline \multicolumn{4}{|l|}{ Biochemical traits } \\
\hline Fasting plasma glucose, mg/dl & $100.28(23.37)$ & $132.7(38.71)$ & $98.73(23.12)$ \\
\hline Total cholesterol, mg/dl & $196.62(36.79)$ & $188.9(40.78)$ & $212.18(43.92)$ \\
\hline HDL cholesterol, mg/dl & $51.65(12.72)$ & $49.01(11.9)$ & $48.44(13.8)$ \\
\hline Triglyceride, mg/dl & $143.83(95.78)$ & $159.51(119.82)$ & $176.4(114.14)$ \\
\hline LDL cholesterol, mg/dl & $116.23(34.5)$ & $108.02(37.21)$ & $128.47(42.28)$ \\
\hline \multicolumn{4}{|l|}{ Lifestyle factors } \\
\hline $\begin{array}{l}\text { Smoking status: Never/Quit/Current, } \\
\text { No. (\%) }\end{array}$ & $\begin{array}{l}10,979(67.3 \%) / 3497(21.4 \%) / \\
1833(11.2 \%)\end{array}$ & $\begin{array}{l}3221(60.6 \%) / 1266(23.8 \%) / \\
827(15.6 \%)\end{array}$ & $\begin{array}{l}13,889(66.9 \%) / 3849(18.5 \%) / \\
3032(14.6 \%)\end{array}$ \\
\hline $\begin{array}{l}\text { Drinking status: Never/Quit/Current, } \\
\text { No. (\%) }\end{array}$ & $7965(48.8 \%) / 760(4.7 \%) / 7584(46.5 \%)$ & $2629(49.5 \%) / 325(6.1 \%) / 2360(44.4 \%)$ & $\begin{array}{l}\text { 10,592 (51\%)/ } 891(4.3 \%) / \\
9287(44.7 \%)\end{array}$ \\
\hline Exercise status: No/Yes, No. (\%) & $7924(48.6 \%) / 8385(51.4 \%)$ & $2633(49.5 \%) / 2681(50.5 \%)$ & $10,040(48.3 \%) / 10,730(51.7 \%)$ \\
\hline
\end{tabular}

Continuous variables are presented as mean (SD). Categorical variables are presented as No. (\%)

10 suggestive loci. The leading SNP of CAD in HTN (17q25.3/CBX8-CBX4 rs1550676) showed the strongest association $\left(P=6.37 \times 10^{-9}\right)$ and a high odds ratio (OR) of 2.607 (95\% CI 1.886-3.602), and a total of 4 significant SNPs were clustered in the locus.

\section{GWAS results of CVDs in DM}

The GWAS of DM-CAD (Fig. 2d) showed 18 suggestive loci and that of DM-IS (Fig. 2e) showed 1 novel significant locus (4q32.2) and 41 suggestive loci. In the GWAS of DM-CCD (Fig. 2f), 1 locus, 17q25.3, was again shown to be significant with 21 suggestive loci. The leading SNP of CAD and CCD in DM (17q25.3/RPTOR rs139293840) showed strong associations $\left(P=1.99 \times 10^{-8}\right)$ as well as a high OR (3.511, 95\%CI 2.265-5.444). The leading SNP for IS (4q32.3/MARCH1-LINC01207 rs149608518) also showed significance $\left(P=1.34 \times 10^{-8}\right)$ and a significantly high OR (5.587, 95\%CI 3.086-10.12).

\section{GWAS results of CVDs in DL}

In DL, all 3 GWASs identified genome-wide significant loci: in DL-CAD (Fig. 2g), 1 significant locus (9q22.2) and 24 suggestive loci; in DL-IS (Fig. 2h), 1 significant locus (3p22.1) and 40 suggestive loci; and in DL-CCD (Fig. 2i), 1 significant locus (2p22.2) and 19 suggestive loci. A total of 11 significant SNPs were clustered in 9q22.2/UNQ6494LOC10192784 associated with CAD, the lead SNP being rs79166762 $\left(P=7.78 \times 10^{-9}, \mathrm{OR}=2.282\right.$ [95\% CI 1.7243.019]). 3p22.1/ULK4 rs3897976 showed significance for IS $\left(P=2.97 \times 10^{-8}, \mathrm{OR}=2.162[95 \%\right.$ CI $\left.1.646-2.84]\right)$ and 2p22.2/CYP1B1-CYP1B1-AS1 rs189668056 for CCD $\left(P=4.24 \times 10^{-8}, \mathrm{OR}=2.027\right.$ [95\% CI 1.574-2.609]).

\section{Discussion}

Despite large-scale GWASs providing robust evidence for genetic variants influencing metabolic pathways and CVDs, [19-24] it is unclear whether genetic variants exist for CVD within the basis of individual chronic metabolic diseases. The aim of our study was to identify genetic variations associated with CVD among individuals with HTN, DM, and DL, respectively; for which we found varied meaningful loci for CVD.

In HTN, locus 17q25.3 [CBX8-CBX4] showed the highest significance for CAD; intriguingly, 17q25.3 was also found to uniformly show suggestive associations for CAD in both DM and DL, which shall be discussed in detail later. Candidate genes for HTN-CAD risk include $C B X 2 / 4 / 8, G A A, C A R D 14$, and SLC26A11. 


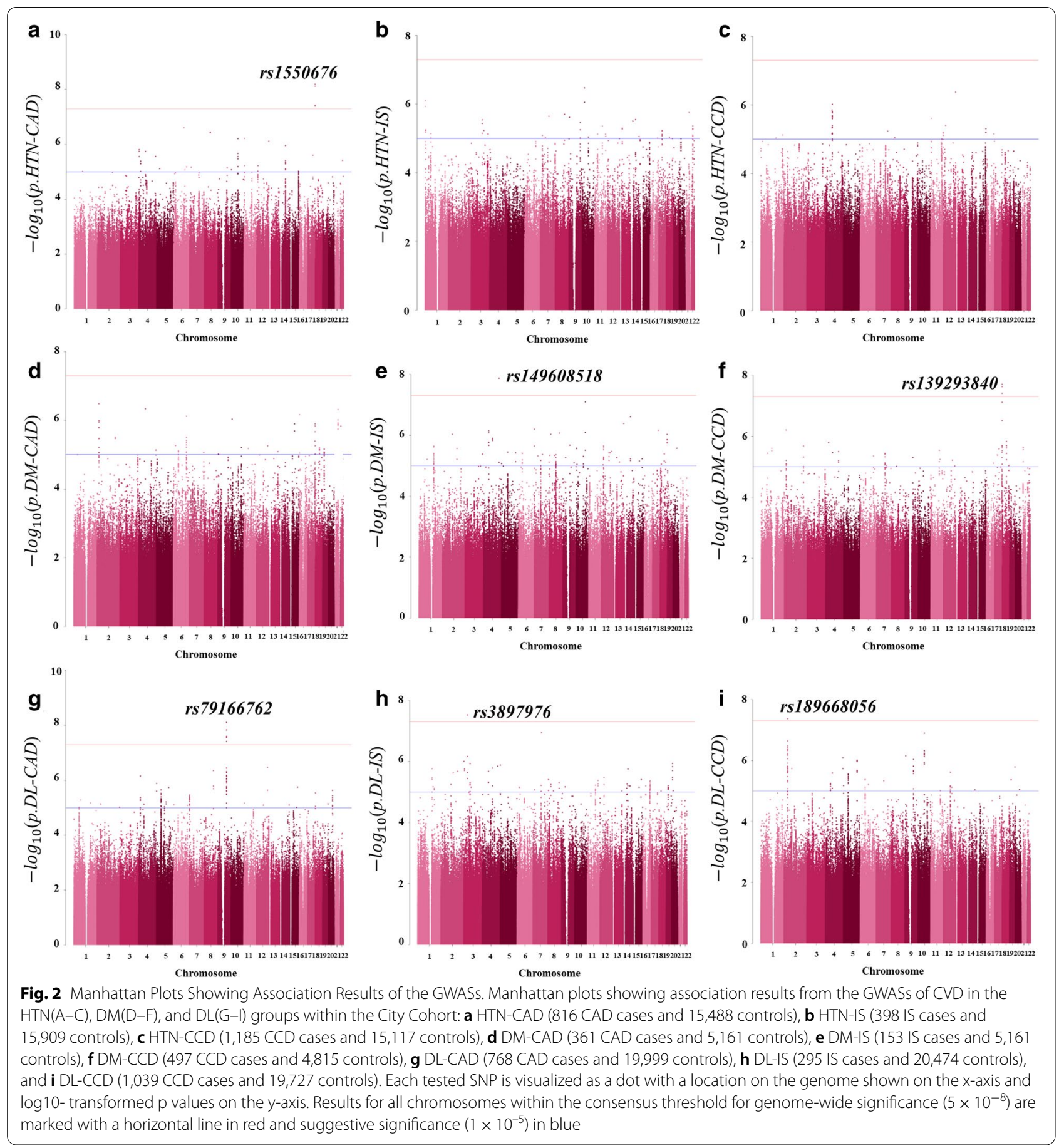

The $C B X$ gene family, a recent research interest in the field of long noncoding RNAs, has been associated with CVD and atherosclerosis in multiple studies [25, 26]. GAA gene expression has been found to be specifically increased in CAD patients than in controls [27]. CARD14, which is mainly associated with psoriasis (ongoing clinical studies suggest increased risk of CVDs in psoriasis) and auto-inflammatory disorders, acts as a pro-inflammatory gene by affecting $N F-k B$ activation in the IL-17 inflammation pathway. Puig et al. [28] found that expression of genes such as CARD14 classified human atherosclerotic plaque by relative inflammation status. SLC26A11, a product of the SLC26A family of anion transporters, has been associated with both 


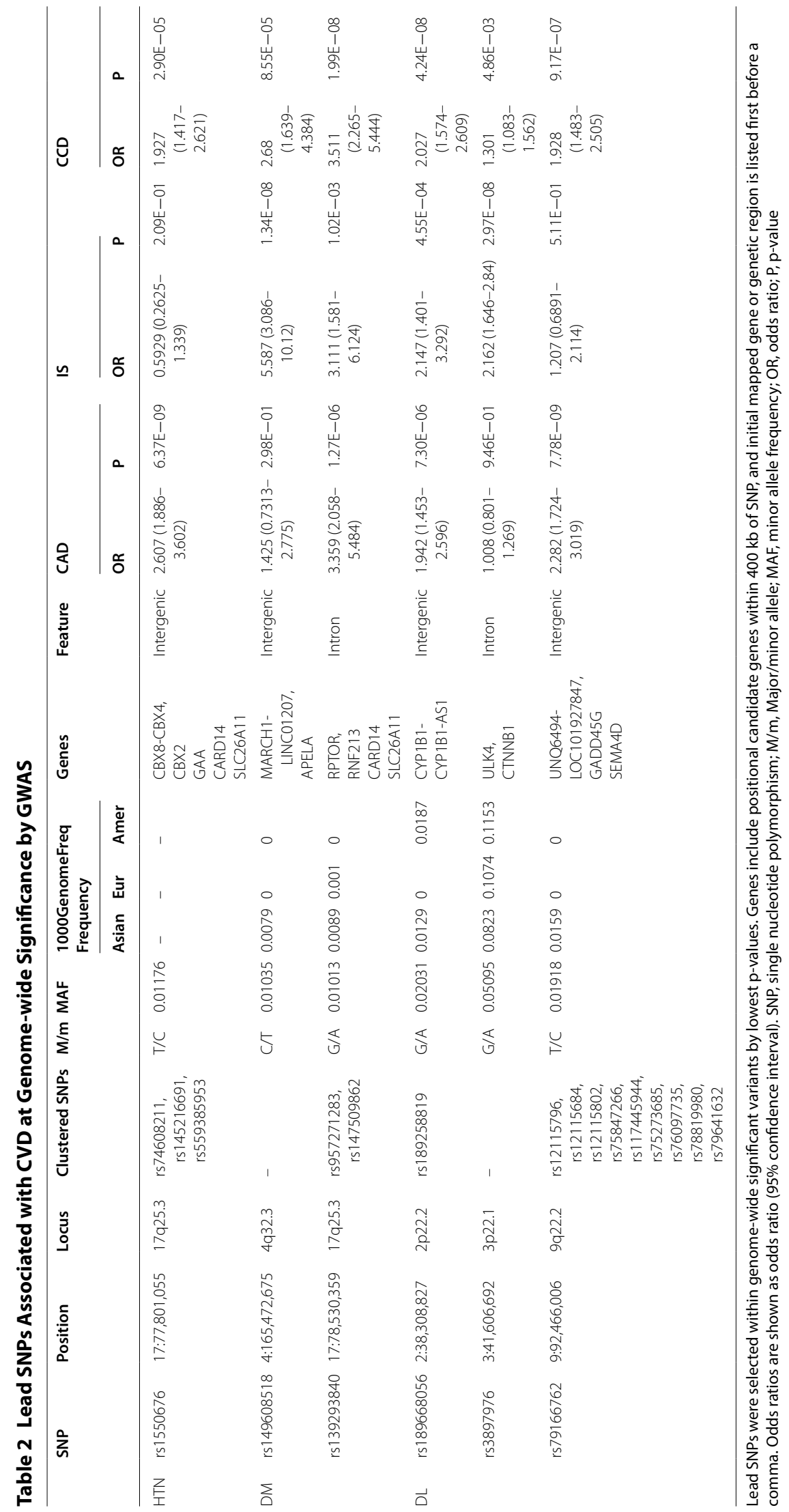


cardiovascular and cerebrovascular disease; notably, it was postulated that $S L C 26 A 11$ tagged multiple variants of RNF213 associated with young-onset IS [29], and SLC26A11 was detected from blood samples of patients with ST-elevation MI [30].

Interestingly, CARD14 and SLC26A11 in the region of $17 \mathrm{q} 25.3$ were located within $400 \mathrm{~kb}$ of the lead SNP for CCD in DM (17q25.3/RPTOR rs139293840) as well. HTN and DM share common risk factors and frequently co-occur [31]; additionally, CVD is the most common long-term complication of both [6]. The overlapping association of SNPs and genetic loci between HTN and DM support the existence of shared points of regulation for these phenotypes [31].

RPTOR, RNF213, CARD14, and SLC26A11 all bear the potential to be the mechanism behind DM-CCD risk loci 17q25.3 [RPTOR]. RNF213 plays important roles in vascular development, and mutant RNF213 has been shown to reduce angiogenesis and induce endothelial dysfunction [32]. RNF213 has been identified as a susceptibility gene of Moyamoya disease [33], intracranial arterial stenoses [34], and systemic vasculopathy among East Asian populations [33] and CAD in the Japanese population [34]. Recently, the significance of RNF213 and SLC26A11 in Caucasian populations were reported as well [29], which encourages its re-evaluation within other ethnicities. RPTOR is a component of the $m T O R$ pathway, which regulates cell growth in response to nutrient levels by associating with the mammalian target of rapamycin (mTOR) [35]. New insights have been offered into RPTOR and CVD/atherosclerosis; such as evidence that miR-100 exerts anti-angiogenic properties through suppression of $m T O R$, [25] and that inhibition of cardiac GSK-3 $\beta$ during continued myocardial ischemia attenuates ischemia-induced mTORC1 inhibition and increases ischemic injury [35].

Rs139293840 (17q25.3/RPTOR, minor allele frequency (MAF) 0.01) showed substantial risk for DM-CAD (OR 3.36 [2.06-5.48]); although the association was suggestive $\left(P=1.27 \times 10^{-6}\right.$; however, genome-wide significance in DM-CCD, $P=1.99 \times 10^{-8}$ ), this could be due to insufficient statistical power after selecting populations for quality control. Genetic risk factors regarding CAD have usually been found to have only a modest effect, conferring an OR ranging from 1.05 to 1.20 [36]; considering the effect size, further replication studies with larger sample sizes are warranted. Based on genotyping data, the MAF of rs139293840 varies among different ethnic populations, ranging from $0.89 \%$ in Asians to $0.01 \%$ in the population of European ancestry to $0 \%$ in the American population. Thus, the association between rs139293840 and CAD may be more prevalent in the Asian population.
The locus, 4p32.3 [MARCH1-LINC01207] showed significant risk for DM-IS (OR 5.587 [3.086-10.12]) and was located near APELA and MARCH1. APELA is a novel endogenous peptide ligand that activates the APJ receptor axis and is cardioprotective against $\mathrm{HTN}, \mathrm{MI}$, pulmonary arterial hypertension, and heart failure [37, 38]. Recently, studies have shown that the apelin/APJ axis possess neuroprotective effects by inhibiting neuronal apoptosis and improving functional recovery in IS through diverse mechanisms, including suppressing inflammatory responses, modulating autophagy, and promoting angiogenesis [39]. MARCH1 has been identified as a susceptibility locus for MI and HTN in Japanese [40], and regarding IS, was included as 1 of the 31 SNPs significantly associated with IS in an initial EWAS [41].

In DL-CAD risk, locus 9q22.2 [UNQ6494LOC10192784] was significant; Semaphorin 4D (SEMA4D) and Growth arrest and DNA-damage-inducible 45 (GADD45G) are potential candidates. Sema4D is a type I integral membrane glycoprotein expressed by most hematopoietic cells that participate in the pathogenesis of atherosclerosis, which has been identified as an independent risk factor for CHD [42]. GADD45G, of the GADD45 family involved in p38 mitogen-activated protein kinase-dependent cell death, has been linked with several cardiologic studies [43]. Recently, conferred resistance to myocardial ischemic injury and cardiomyocyte apoptosis was found when GADD45G was deleted in mice [44].

Again, the gene-rich locus 17q25.3 was found to be of suggestive association for CAD in DL. Considering the small sample size, we consider this finding to bear significance, which confers on 17q25.3 the status of a common susceptibility locus of CVD in HTN, DM, and DL. It has not been fully elucidated whether these chronic metabolic diseases are part of the causal pathway for CVD or merely comorbid diseases in "common soil". The search has been fraught with controversial findings, as has the presence of overlap in the genetic architecture of metabolic diseases and CVDs. This study provides first probable evidence of a common candidate locus for CVD in the 3 diseases using a GWAS approach.

Locus 3p22.1 [ULK4] of DL-IS risk seems likely to be associated with ULK4; recently, ULK4 was identified by GWAS and brain eQTL to be a susceptibility gene for IS and small vessel stroke in trans-ethnic datasets [45]. However, CTNN1B of the WNT gene family is another possibility. The Wnt signaling pathway contributes to the development of CVDs; both canonical and noncanonical Wnt signaling cascades moderate cell phenotypic modulation of vascular smooth muscles in CVDs [46]. CTNN1B encodes beta-catenin and is essential in maintaining CNS homeostasis [47]; in terms of arteriosclerotic 
effects, CTNN1B was found to harbor a susceptibility variant for MI in the Han population [48]. Recently, expression of vital genes in the Wnt pathway (including CTNN1B) was found to be upregulated in cerebral infarction in rats [46]; it seems plausible that similar findings could be found in humans.

Finally, 2p22.2 [CYP1B1-CYP1B1-AS1] of DL$\mathrm{CCD}$ risk could be associated with Cytochrome $\mathrm{P} 450$ 1B1(CYP1B1), a member of the well-known Cytochrome $\mathrm{P} 450$ family. CYP1B1 is expressed in various tissues, with the highest mRNA expression levels found extrahepatically, such as the heart and brain [49]. Recent findings have shown that $C Y P 1 B 1$ contributes to the development of atherosclerosis, DL, and HTN in rats [50]; demonstrates an association with DM [51]; and has a suggested influence on adipogenesis [49].

\section{Strengths and limitations}

There are limitations to our study. First, replication is lacking, and the findings may not be generalized to cohorts with different ethnicities and races. Second, we investigated the phenotypes of diseases using a patientreported questionnaire and thus could not investigate various CVDs or measurable continuous traits. Third, this work is based on statistical evidence and does not provide functional experimental validation. Potential genetic interactions using biological and mechanical analyses are required to confirm these findings, and additional studies are warranted for replication and validation.

Despite these limitations, our study has several unique strengths. Many studies have demonstrated varied findings regarding the genetic relations between CVD and its risk factors [52-54]. However, most previous studies utilized variants of genome-wide significance or genotyping arrays that focused only on pre-selected loci, and they have not fully utilized genome-wide variation for the estimation of genetic variants of CVD risk [55-57]. Moreover, none of these previous studies have focused on the genetic variants of CVD within the same chronic conditions. To the best of our knowledge, the present study provides novel evidence of genetic associations with CVD specific to individuals with HTN, DM, and DL. Charmet et al. [58] found novel candidates for CAD using a then unprecedented similar study design (434 cases and 3,123 controls), in patients with type 1 diabetes. The barrier of limited statistical power due to sample size may be why such studies are lacking.

Although some of our findings showed low prevalence, the overall effect size is significant with high ORs. Also, the allele frequency may be underestimated due to smaller quality genomic databases of East Asians used in forming the reference panels. Further, cardio-metabolic diseases associated with CVDs have been increasing in prevalence as well as in total medical costs in Asians, with HTN and DM ranking 1st and 2nd among all medical diagnoses in Korea, respectively [59]. Dyslipidemia prevalence has been rapidly increasing up to $38.4 \%$ of all adults, and CVDs account for $1 / 4$ of total mortality of the Korean population according to recent data [60]. Our preliminary findings may be used to improve the identification of individuals with HTN, DM, or DL at high-risk for cardiovascular events or to provide novel targets for potential pharmacologic development. In the era of personalized, preventive medicine with progressively aging populations with chronic cardio-metabolic diseases, further comprehensive analyses of our findings seem highly warranted.

\section{Conclusions}

In conclusion, our GWAS, of unprecedented design, provides several novel insights into the genetic architecture of CVDs. We report 6 loci with genome-wide significant association for CVDs, which harbor numerous potential positional candidate genes, and show substantial CVD risk discrimination in individuals with $\mathrm{HTN}, \mathrm{DM}$, or DL. In addition, we suggest the importance of $17 q 25.3$ as a potential common CAD locus for HTN, DM, and DL.

\section{Supplementary Information}

The online version contains supplementary material available at https://doi. org/10.1186/s12967-021-02751-3.

Additional file 1: Table S1. Baseline Characteristics of the Hypertension Study Population. Table S2. Baseline Characteristics of the Diabetes Mellitus Study Population. Table S3. Baseline Characteristics of the Dyslipidemia Study Population. Figure S1. Quantile-quantile plots with genomic inflation values. Figure S2. Regional association plots for lead SNPS, $\mathbf{A}$ HTN-CAD B DM-IS C DM-CCD D DL-CAD E DL-CCD F DL-IS.

Additional file 2: Table S4. Original GWAS results from discovery for association with CVD in HTN. Table S5. Original GWAS results from discovery for association with CVD in DM. Table S6. Original GWAS results from discovery for association with CVD in DL.

\section{Abbreviations}

BMI: Body mass index; CAD: Coronary artery disease; IS: Ischemic stroke; CVD: Cardiovascular disease; GWAS: Genome-wide association study; HDL: High-density lipoprotein; LDL: Low-density lipoprotein; SBP: Systolic blood pressure; SNP: Single-nucleotide polymorphism; DM: Type 2 diabetes mellitus; DL: Dyslipidemia; KoGES: Korean Genome and Epidemiology Study; NCEP-ATP III: National Cholesterol Education Program- Adult Treatment Panel III; PAH:

Pulmonary artery hypertension; HF: Heart failure.

\section{Acknowledgements}

This study was conducted with bioresources graciously provided by the National Biobank of Korea, the Centers of Disease Control and Prevention, Republic of Korea (2019-059).

\section{Authors' contributions}

JWL and KWH conceptualized the research; JWL, KWH, YS and JEC designed the work; YS, JEC, YJK, HJC, JOK and DHP acquired and analyzed the data; YS, 
JEC, YJK, HJC, JMP and SJK interpreted the results. All authors contributed to the drafting and critical revision of the manuscript. All authors read and approved the final manuscript.

\section{Funding}

This study was supported by the Bio and Medical Technology Development Program through the National Research Foundation of Korea funded by the Ministry of Science, ICT, and Future Planning [NRF2018R1D1A1B07049223] and the Technology Innovation Program [20002781, A Platform for Prediction and Management of Health Risk Based on Personal Big Data and Lifelogging] funded by the Ministry of Trade, Industry \& Energy (MOTIE, Korea).

\section{Availability of data and materials}

The datasets used and/or analyzed during the current study are available from the corresponding authors on reasonable request.

\section{Ethics approval and consent to participate}

All participants voluntarily signed an informed consent form before KoGES, and the study protocol was approved by the Institutional Review Boards (IRB) of the institutions that participated in KoGES. This current study was performed in accordance with the Declaration of Helsinki and approved by the IRB of Theragen Etex (Approval Numbers: 700062-20190819-GP-006-02).

\section{Consent for publication}

Not applicable.

\section{Competing interests}

The authors declare that they have no competing interests.

\section{Author details}

${ }^{1}$ Department of Family Medicine, Gangnam Severance Hospital, Yonsei University College of Medicine, 211, Eonju-ro, Gangnam-gu, Seoul 06273, Korea. 2 Healthcare R\&D Division, Theragen Bio Co., Ltd., Gwanggyo-ro 145, Suwon-si, Gyeonggi-do 16229, Republic of Korea. ${ }^{3}$ Department of Family Medicine, Yongin Severance Hospital, Yonsei University College of Medicine, 363, Dongbaekjukjeon-daero, Giheung-gu, Yongin-si 16995, Gyeonggi-do, Korea. ${ }^{4}$ Division of Cardiology, Severance Cardiovascular Hospital, Yonsei University College of Medicine, 50-1, Yonsei-ro, Seodaemun-gu, Seoul 03722, Korea.

Received: 27 November 2020 Accepted: 11 February 2021 Published online: 25 February 2021

\section{References}

1. Rizvi S, Kamran H, Salciccioli L, Saiful F, Lafferty J, Lazar JM. Relation of the ankle brachial index to left ventricular ejection fraction. Am J Cardiol. 2010;105:129-32.

2. Rhee EJ. Prevalence and current management of cardiovascular risk factors in Korean adults based on fact sheets. Endocrinol Metab (Seoul). 2020;35:85-94.

3. Shin H-Y, Lee J-Y, Song J, Lee S, Lee J, Lim B, Kim H, Huh S. Cause-ofdeath statistics in the Republic of Korea, 2014. J Korean Med Assoc. 2016;59:221-32.

4. Hedayatnia M, Asadi Z, Zare-Feyzabadi R, Yaghooti-Khorasani M, Ghazizadeh H, Ghaffarian-Zirak R, Nosrati-Tirkani A, Mohammadi-Bajgiran M, Rohban M, Sadabadi F, et al. Dyslipidemia and cardiovascular disease risk among the MASHAD study population. Lipids Health Dis. 2020;19:42.

5. Ettehad D, Emdin CA, Kiran A, Anderson SG, Callender T, Emberson J, Chalmers J, Rodgers A, Rahimi K. Blood pressure lowering for prevention of cardiovascular disease and death: a systematic review and meta-analysis. Lancet. 2016;387:957-67.

6. Einarson TR, Acs A, Ludwig C, Panton UH. Prevalence of cardiovascular disease in type 2 diabetes: a systematic literature review of scientific evidence from across the world in 2007-2017. Cardiovasc Diabetol. 2018;17:83.

7. Darroudi S, Saberi-Karimian M, Tayefi M, Arekhi S, Motamedzadeh Torghabeh A, Seyedzadeh Sani SMR, Moohebati M, Heidari-Bakavoli A, Ebrahimi M, Azarpajouh MR, et al. Prevalence of combined and noncombined dyslipidemia in an Iranian population. J Clin Lab Anal. 2018;32:e22579.
8. Borén J, Chapman MJ, Krauss RM, Packard CJ, Bentzon JF, Binder CJ, Daemen MJ, Demer LL, Hegele RA, Nicholls SJ, et al. Low-density lipoproteins cause atherosclerotic cardiovascular disease: pathophysiological, genetic, and therapeutic insights: a consensus statement from the European Atherosclerosis Society Consensus Panel. Eur Heart J. 2020;41:2313-30.

9. Libby P, Ridker Paul M, Maseri A. Inflammation and atherosclerosis. Circulation. 2002;105:1135-43.

10. Thrainsdottir IS, Aspelund T, Thorgeirsson G, Gudnason V, Hardarson T, Malmberg K, Sigurdsson G, Rydén L. The association between glucose abnormalities and heart failure in the population-based Reykjavík Study. Diabetes Care. 2005;28:612.

11. Miller M. Dyslipidemia and cardiovascular risk: the importance of early prevention. QJM Monthly J Assoc Phys. 2009;102:657-67.

12. Lloyd-Jones DM, Nam BH, D’Agostino RB Sr, Levy D, Murabito JM, Wang TJ, Wilson PW, O'Donnell CJ. Parental cardiovascular disease as a risk factor for cardiovascular disease in middle-aged adults: a prospective study of parents and offspring. JAMA. 2004;291:2204-11.

13. Rankinen T, Sarzynski Mark A, Ghosh S, Bouchard C. Are there genetic paths common to obesity, cardiovascular disease outcomes, and cardiovascular risk factors? Circ Res. 2015;116:909-22.

14. McPherson R, Tybjaerg-Hansen A. Genetics of coronary artery disease. Circ Res. 2016;118:564-78.

15. Kim Y, Han BG. Ko GESg: cohort profile: the korean genome and epidemiology study (KoGES) Consortium. Int J Epidemiol. 2017;46:e20.

16. Chang CC, Chow CC, Tellier LCAM, Vattikuti S, Purcell SM, Lee JJ. Secondgeneration PLINK: rising to the challenge of larger and richer datasets. GigaScience. 2015;4:s13742-015.

17. Panagiotou OA, loannidis JP. Genome-Wide Significance P: What should the genome-wide significance threshold be? Empirical replication of borderline genetic associations. Int J Epidemiol. 2012;41:273-86.

18. Pruim RJ, Welch RP, Sanna S, Teslovich TM, Chines PS, Gliedt TP, Boehnke M, Abecasis GR, Willer CJ. LocusZoom: regional visualization of genome-wide association scan results. Bioinformatics (Oxford, England). 2010;26:2336-7.

19. Wang Y, Wang JG. Genome-wide association studies of hypertension and several other cardiovascular diseases. Pulse (Basel). 2019;6:169-86.

20. Das S, Reddy MA, Natarajan R. Role of epigenetic mechanisms regulated by enhancers and long noncoding RNAs in cardiovascular disease. Curr Opin Cardiol. 2020;35:234-41.

21. Goodarzi MO, Rotter Jl. Genetics insights in the relationship between type 2 diabetes and coronary heart disease. Circ Res. 2020;126:1526-48.

22. Lotta LA, Stewart ID, Sharp SJ, Day FR, Burgess S, Luan J, Bowker N, Cai L, Li C, Wittemans LBL, et al. Association of genetically enhanced lipoprotein lipase-mediated lipolysis and low-density lipoprotein cholesterollowering alleles with risk of coronary disease and type 2 diabetes. JAMA Cardiol. 2018:3:957-66.

23. Mannino GC, Pezzilli S, Averta C, Fuoco A, Spiga R, Mancuso E, Di Fatta C, Perticone F, Prudente S, Trischitta V, et al. A functional variant of the dimethylarginine dimethylaminohydrolase-2 gene is associated with myocardial infarction in type 2 diabetic patients. Cardiovasc Diabetol. 2019:18:102.

24. Zheng Q, Jiang J, Huo Y, Chen D. Genetic predisposition to type 2 diabetes is associated with severity of coronary artery disease in patients with acute coronary syndromes. Cardiovasc Diabetol. 2019;18:131.

25. Fasolo F, Di Gregoli K, Maegdefessel L, Johnson JL. Non-coding RNAs in cardiovascular cell biology and atherosclerosis. Cardiovasc Res. 2019;115:1732-56.

26. Hennessy EJ. Cardiovascular disease and long noncoding RNAs: tools for unraveling the mystery Lnc-ing RNA and phenotype. Circ Cardiovasc Genet. 2017;10:e001556.

27. Zhang J, Ma L, Zhang J, Huang J, Wei G, Liu L, Zhang J, Yan B. Altered expression of lysosomal hydrolase, acid alpha-glucosidase, gene in coronary artery disease. Coron Artery Dis. 2016;27:104-8.

28. Puig O, Yuan J, Stepaniants S, Zieba R, Zycband E, Morris M, Coulter S, Yu X, Menke J, Woods J, et al. A gene expression signature that classifies human atherosclerotic plaque by relative inflammation status. Circ Cardiovasc Genet. 2011;4:595-604.

29. Lorenzo-Betancor O, Blackburn PR, Farrugia L, Soto-Beasley Al, Walton RL, Edwards E, Tawk RG, Klee EW, Freeman WD, Miller D, et al. RNF213 variation, a broader role in neurovascular disease in Caucasian and Japanese populations. bioRxiv 2020:2020.2003.2031.013078. 
30. Guan YZ, Yin RX, Zheng PF, Deng GX, Liu CX, Wei BL. Potential molecular mechanism of ACE gene at different time points in STEMI patients based on genome-wide microarray dataset. Lipids Health Dis. 2019;18:184.

31. Ibanez L, Heitsch L, Dube U, Farias FHG, Budde J, Bergmann K, Davenport R, Bradley J, Carrera C, Kinnunen J, et al. Overlap in the genetic architecture of stroke risk, early neurological changes, and cardiovascular risk factors. Stroke. 2019;50:1339-45.

32. Kotani Y, Morito D, Yamazaki S, Ogino K, Kawakami K, Takashima S, Hirata $\mathrm{H}$, Nagata K. Neuromuscular regulation in zebrafish by a large AAA+ ATPase/ubiquitin ligase, mysterin/RNF213. Sci Rep. 2015;5:16161.

33. Bang OY, Chung JW, Kim DH, Won HH, Yeon JY, Ki CS, Shin HJ, Kim JS, Hong SC, Kim DK, Koizumi A. Moyamoya disease and spectrums of RNF213 vasculopathy. Transl Stroke Res. 2019;11:580-9.

34. Morimoto T, Mineharu Y, Ono K, Nakatochi M, Ichihara S, Kabata R, Takagi Y, Cao Y, Zhao L, Kobayashi H, et al. Significant association of RNF213 p.R4810K, a moyamoya susceptibility variant, with coronary artery disease. PLoS ONE. 2017;12:e0175649.

35. Sciarretta S, Forte M, Frati G, Sadoshima J. New insights into the role of mTOR signaling in the cardiovascular system. Circ Res. 2018;122:489-505.

36. Shah SJ, Arnett DK. Cardiovascular genetics and genomics in clinical practice. Berlin: Springer Publishing Company; 2014.

37. Zhong JC, Zhang ZZ, Wang W, McKinnie SMK, Vederas JC, Oudit GY. Targeting the apelin pathway as a novel therapeutic approach for cardiovascular diseases. Biochim Biophys Acta Mol Basis Dis. 2017;1863:1942-50.

38. Griffiths PR, Lolait SJ, Bijabhai A, O'Carroll-Lolait A, Paton JFR, O'Carroll AM. Increased apelin receptor gene expression in the subfornical organ of spontaneously hypertensive rats. PLoS ONE. 2020;15:e0231844.

39. Tian $Y$, Chen $R$, Jiang Y, Bai B, Yang T, Liu $H$. The protective effects and mechanisms of apelin/APJ system on ischemic stroke: a promising therapeutic target. Front Neurol. 2020;11:75.

40. Yamada Y, Kato K, Oguri M, Horibe H, Fujimaki T, Yasukochi Y, Takeuchi I, Sakuma J. Identification of 13 novel susceptibility loci for early-onset myocardial infarction, hypertension, or chronic kidney disease. Int J Mol Med. 2018;42:2415-36.

41. Yamada Y, Kato K, Oguri M, Horibe H, Fujimaki T, Yasukochi Y, Takeuchi I, Sakuma J. Identification of nine genes as novel susceptibility loci for early-onset ischemic stroke, intracerebral hemorrhage, or subarachnoid hemorrhage. Biomed Rep. 2018;9:8-20.

42. Gong H, Lyu X, Li S, Chen R, Hu M, Zhang X. sSema4D levels are increased in coronary heart disease and associated with the extent of coronary artery stenosis. Life Sci. 2019;219:329-35.

43. Iwahana T, Okada S, Kanda M, Oshima M, Iwama A, Matsumiya G, Kobayashi Y. Novel myocardial markers GADD45G and NDUFS5 identified by RNA-sequencing predicts left ventricular reverse remodeling in advanced non-ischemic heart failure: a retrospective cohort study. BMC Cardiovasc Disord. 2020;20:116.

44. Lucas A, Mialet-Perez J, Daviaud D, Parini A, Marber MS, Sicard P. Gadd45 gamma regulates cardiomyocyte death and post-myocardial infarction left ventricular remodelling. Cardiovasc Res. 2015;108:254-67.

45. Zhao $\mathrm{S}$, Jiang $\mathrm{H}$, Liang $\mathrm{ZH}$, Ju H. Integrating multi-omics data to identify novel disease genes and single-neucleotide polymorphisms. Front Genet. 2019;10:1336.

46. Sun JD, Li XM, Liu JL, Li J, Zhou H. Effects of miR-150-5p on cerebral infarction rats by regulating the Wnt signaling pathway via p53. Eur Rev Med Pharmacol Sci. 2020;24:3882-91.
47. Tran KA, Zhang X, Predescu D, Huang X, Machado RF, Gothert JR, Malik $A B$, Valyi-Nagy $T$, Zhao YY. Endothelial beta-catenin signaling is required for maintaining adult blood-brain barrier integrity and central nervous system homeostasis. Circulation. 2016;133:177-86.

48. Tao J, Wang YT, Abudoukelimu M, Yang YN, Li XM, Xie X, Chen BD, Liu F, $\mathrm{He} \mathrm{CH}$, Li HY, Ma YT. Association of genetic variations in the Wnt signaling pathway genes with myocardial infarction susceptibility in Chinese Han population. Oncotarget. 2016;7:52740-50.

49. Li F, Zhu W, Gonzalez FJ. Potential role of CYP1B1 in the development and treatment of metabolic diseases. Pharmacol Ther. 2017;178:18-30.

50. Song CY, Ghafoor K, Ghafoor HU, Khan NS, Thirunavukkarasu S, Jennings BL, Estes AM, Zaidi S, Bridges D, Tso P, et al. Cytochrome P450 1B1 contributes to the development of atherosclerosis and hypertension in apolipoprotein E-deficient mice. Hypertension. 2016;67:206-13.

51. Elfaki I, Mir R, Almutairi FM, Duhier FMA. Cytochrome P450: polymorphisms and roles in cancer, diabetes and atherosclerosis. Asian Pac J Cancer Prev. 2018;19:2057-70.

52. Wu C, Gong Y, Yuan J, Gong H, Zou Y, Ge J. Identification of shared genetic susceptibility locus for coronary artery disease, type 2 diabetes and obesity: a meta-analysis of genome-wide studies. Cardiovasc Diabetol. 2012;11:68

53. Adams JN, Raffield LM, Freedman BI, Langefeld CD, Ng MC, Carr JJ, Cox AJ, Bowden DW. Analysis of common and coding variants with cardiovascular disease in the Diabetes Heart Study. Cardiovasc Diabetol. 2014;13:77.

54. Beaney KE, Ward CE, Bappa DA, McGale N, Davies AK, Hirani SP, Li K, Howard P, Vance DR, Crockard MA, et al. A 19-SNP coronary heart disease gene score profile in subjects with type 2 diabetes: the coronary heart disease risk in type 2 diabetes (CoRDia study) study baseline characteristics. Cardiovasc Diabetol. 2016;15:141.

55. Fall T, Gustafsson S, Orho-Melander M, Ingelsson E. Genome-wide association study of coronary artery disease among individuals with diabetes: the UK Biobank. Diabetologia. 2018;61:2174-9.

56. LeBlanc M, Zuber V, Andreassen BK, Witoelar A, Zeng L, Bettella F, Wang Y, McEvoy LK, Thompson WK, Schork AJ, et al. Identifying novel gene variants in coronary artery disease and shared genes with several cardiovascular risk factors. Circ Res. 2016;118:83-94.

57. Ji LD, Hu SP, Li JY, Yao BB, Shen QJ, Xu J. Shared genetic etiology of hypertension and stroke: evidence from bioinformatics analysis of genomewide association studies. J Hum Hypertens. 2017;32:34-9.

58. Charmet R, Duffy S, Keshavarzi S, Gyorgy B, Marre M, Rossing P, McKnight AJ, Maxwell AP, Ahluwalia TVS, Paterson AD, et al. Novel risk genes identified in a genome-wide association study for coronary artery disease in patients with type 1 diabetes. Cardiovasc Diabetol. 2018;17:61.

59. KCDC: 2019 HTN/DM Practice Guidelines; 2019.

60. (KSoLA) KSoLaA: Dyslipidemia Fact Sheets in Korea, 2020. 2020.

\section{Publisher's Note}

Springer Nature remains neutral with regard to jurisdictional claims in published maps and institutional affiliations.

Ready to submit your research? Choose BMC and benefit from

- fast, convenient online submission

- thorough peer review by experienced researchers in your field

- rapid publication on acceptance

- support for research data, including large and complex data types

- gold Open Access which fosters wider collaboration and increased citations

- maximum visibility for your research: over $100 \mathrm{M}$ website views per year

At BMC, research is always in progress.

Learn more biomedcentral.com/submissions 